\title{
Desarrollo de la Aleta Caudal del Salmón (Salmo salar)
}

\author{
Salmon Caudal Fin Development (Salmo salar)
}

\author{
Mariana Rojas ${ }^{*, * *}$; Mario Pellón" \& Mariano del Sol ${ }^{*, * * *, * * * *}$
}

\begin{abstract}
ROJAS, M.; PELLÓN, M. \& DEL SOL, M. Desarrollo de la aleta caudal del salmón (Salmo salar). Int. J. Morphol., 33(2):514-521, 2015 .
\end{abstract}

RESUMEN: Las patologías y traumas de la aleta caudal afectan la natación, dificultan la alimentación y la eficiencia de escape de los peces, además aumentan la susceptibilidad a las infecciones bacterianas y fúngicas. Los salmones adultos pueden regenerar rápida y completamente su aleta si esta es amputada. Sin embargo, se han reportado en el sur de Chile, alevines que expresan defectos anatómicos en la aleta caudal asociados a un alto índice de mortalidad donde no ocurre regeneración. Existen múltiples estudios sobre la aleta caudal de peces adultos pero esta descripción no concuerda con la morfología de la fase de alevín. Nuestro objetivo es describir la anatomía e histología de la aleta caudal del salmón de $15 \mathrm{~mm}, 30 \mathrm{~mm}$ y $60 \mathrm{~mm}$ para facilitar el diagnóstico de las patologías tempranas de la aleta caudal. Se trabajó con 60 salmones divididos en tres grupos de 20 en etapas de 15, 30 y 60 mm. Diez salmones de cada grupo fueron procesados con técnicas anatómicas de Hanken \& Wassersug . Otros 10 alevines de cada grupo fueron procesados mediante técnicas H\&E/azul de Alcian pH 2,5: para glicosaminoglicanos y técnica Histoquímica Picrosirius de Junqueira para colágeno I y III. Al momento de la eclosión de los peces (grupo 1) la aleta caudal no tiene su forma definitiva pero ha iniciado la formación de lepidotriquias. En el grupo 2, la aleta caudal comprende entre 19-20 lepidotriquias y se constituyen dos lóbulos uno dorsal y otro ventral, ambos bajo la notocorda. Los rayos de cada lóbulo crecen más rápido que los rayos que se encuentran entre los lóbulos y se forma un surco entre ellos. En el grupo 3 se observa claramente la aleta bilobulada, se mantienen 19 lepidotriquias que ahora están en proceso de calcificación. Cada lepidotriquia crece distalmente mediante la formación de articulaciones y segmentos. En el grupo 2 se consignó un promedio de 4-5 articulaciones por lepidotriquia y en el grupo 3 han aumentado a 6-10 articulaciones. Esta descripción de la aleta del alevín normal facilita el diagnóstico de la aleta deformada y aporta conocimientos para comparar el desarrollo ontogenético con las fases de la regeneración después de la amputación de la aleta caudal.

PALABRAS CLAVE: Aleta caudal; Lepidotriquias; Alevín; Salmón; Desarrollo; Salmo salar.

\section{INTRODUCCIÓN}

Las patologías y traumas de la aleta caudal afectan la natación, dificultan la alimentación y la eficiencia de escape de los peces, además aumentan la susceptibilidad a la infecciones bacterianas y fúngicas (Böckelmann et al., 2010) Los teleósteos tales como el pez cebra tienen la capacidad de regenerar rápida y completamente su aleta si esta es amputada (Marí-Beffa et al., 1999; Marí-Beffa \& Murciano, 2010; Rolland-Lagan et al., 2012). Debido a esta eficiencia la aleta caudal de los peces teleósteos se ha convertido en un modelo para el estudio de los mecanismos moleculares que regulan la regeneración debido a su bioestructura simple y accesibilidad a la amputación (Stoick-Cooper et al., 2007). Se han reportado en el sur de Chile, alevines de salmón que expresan defectos anatómicos en la aleta caudal asociados a un alto índice de mortalidad donde no ocurre regeneración y cuyo diagnóstico no es claro. Los estudios descriptivos sobre la morfología de la aleta caudal de salmón adulto (Becerra et al., 1983, 1996) no concuerdan con la morfología de la fase de alevín, por lo tanto se requieren nuevos estudios circunscritos a esta etapa de desarrollo temprano. Nuestros objetivos fueron: 1) describir la anatomía de la aleta caudal del salmón en tres etapas del desarrollo: A los 15 $\mathrm{mm}, 30 \mathrm{~mm}$ y $60 \mathrm{~mm}, 2$ ) reconocer las características histológicas de los rayos o lepidotriquias y de los interrayos, para facilitar el diagnóstico de las patologías de la aleta caudal, como también para entregar mayor información que nos permita comparar el desarrollo ontogenético del alevín con las etapas de regeneración en salmón.

* Programa de Doctorado en Cs. Morfológicas, Universidad de La Frontera, Temuco, Chile.

** Programa de Anatomía y Biología del Desarrollo, ICBM, Facultad de Medicina, Universidad de Chile, Santiago, Chile.

*** Centro de Excelencia en Investigaciones Morfológicas y Quirúrgicas - CEMyQ, Universidad de La Frontera, Temuco, Chile.

***** Centro de Investigación en Ciencias Biomédicas, Universidad Autónoma de Chile, Temuco, Chile. 


\section{MATERIAL Y MÉTODO}

Se trabajó con 60 salmones divididos en tres grupos. Grupo I: 20 alevines recién eclosionados de $15 \mathrm{~mm}$. Grupo II: 20 alevines de $30 \mathrm{~mm}$ de LAC. Grupo III: 20 salmones de $60 \mathrm{~mm}$ de LAC. Todo el procedimiento fue realizado según las normas bioéticas de la Facultad de Medicina, Universidad de La Frontera, para la experimentación con animales de laboratorio.

Los salmones fueron eutanasiados por exposición a $5 \%$ Benzocaina diluida en agua (Kalmagin 20®, Farquímica) y procesados con técnicas anatómicas de diafanización y tinción para cartílago y hueso de Hanken \& Wassersug (1981). 10 alevines de cada grupo, fueron sometidos a digestión proteica (tripsina) y expuestos al colorante Alizarina que tiñe de color rojo las sales de calcio presentes en los tejidos osificados y al colorante Azul de Alcián, que tiñe azul los glicosaminoglicanos. Otros 10 alevines fueron procesados para microscopía, fueron incluidos en parafina sólida seccionados en cortes sagitales seriados de $5 \mu \mathrm{m}$ de espesor, y procesados para microscopía con las siguientes técnicas 1) H \& E/azul de Alcian pH 2,5: permite observar la disposición general de los tejidos y el depósito de glicosaminoglicanos. 2) Técnica Histoquímica Picrosirius de Junqueira (Junqueira et al., 1979), que genera coloración diferencial entre los distintos tipos de fibras colágenas, en base a la birrefringencia específica de la luz, que cada tipo de fibra produce al ser incidida por un haz de luz polarizada. Así, debido al grosor y organización de cada fibra colágena, las fibras tipo I presentan birrefringencia de coloración rojiza, mientras que el colágeno tipo III se muestra de coloración verdosa (Junqueira et al.). Los tejidos fueron observados en microscopio de luz (Axiostar Plus, Zeiss) que cuenta con un sistema de polarización para visualizar la tinción diferencial del colorante picrosirius y una cámara incorporada (Canon Power Shot A640). Se utilizo el programa Axiostar para realizar mediciones lineales de las lepidotriquias de mayor longitud. Los valores obtenidos para las variables cuantitativas se describen a través de la media aritmética y la desviación estándar.

\section{RESULTADOS}

En la Tabla I se resumen las principales características de los tres grupos de peces estudiados, tales como cambios morfológicos, longitud, número de segmentaciones, de bifurcaciones de las lepidotriquias, aspectos histológicos y de tinción.

Al momento de la eclosión de los peces (grupo I) se observa la presencia de la aleta mediana que es un indicador de las etapas tempranas del desarrollo (Fig. 1). En el grupo

Tabla I. Características generales y cambios de la aleta caudal de salmón durante el desarrollo post-eclosional.

\begin{tabular}{|c|c|c|c|}
\hline Carácter & Grupo A. 15 mm & Grupo B. 30mm & Grupo C. 60 mm \\
\hline Características del pez & $\begin{array}{l}\text { Recién eclosionado, transpa- } \\
\text { rente, saco vitelino grande. } \\
\text { Se mantiene inmóvil. }\end{array}$ & $\begin{array}{l}\text { Larva con saco vitelino en } \\
\text { involución. Presenta movimien- } \\
\text { tos natatorios. Pierde transpa- } \\
\text { rencia }\end{array}$ & $\begin{array}{l}\text { La piel presenta manchas a } \\
\text { ambos lados del cuerpo (par). }\end{array}$ \\
\hline Aleta mediana & Presente & Involucionando & Ausente \\
\hline Aleta caudal & Ausencia de lóbulos & Dos lóbulos simétricos & Dos lóbulos simétricos \\
\hline Nro de lepidotriquias & $11+2$ rayos & $18+1$ rayos & $18+1$ rayos \\
\hline $\begin{array}{l}\text { Longitud de las lepidotriquias } \\
\text { más grandes en mm }\end{array}$ & $18+2$ & $43+0,5$ & $60+0,5$ \\
\hline $\begin{array}{l}\text { Longitud lepidotriquias mas } \\
\text { cortas (medial es) }\end{array}$ & $18+2$ & $40+0,2$ & $56+0,4$ \\
\hline Segmentaciones & 0 & 6 & 10 \\
\hline Bifurcaciones & No & $\mathrm{Si}$ & $\mathrm{Si}$ \\
\hline Histología & 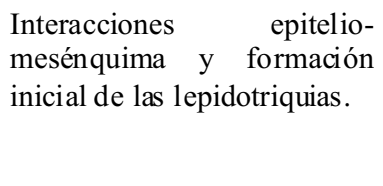 & $\begin{array}{l}\text { Las lepidotriquias presentan un } \\
\text { blastema fuertemente Alcián } \\
\text { positivo y s e envuelven por } \\
\text { tejdo osteoide, que deterrmina } \\
\text { su aumento en espesor. }\end{array}$ & $\begin{array}{l}\text { La calcificación de hueso } \\
\text { progresa desde la región pro- } \\
\text { ximal a la distal. }\end{array}$ \\
\hline $\begin{array}{l}\text { Características observables } \\
\text { con técnica de Hanken \& } \\
\text { Wassersug (1981). }\end{array}$ & Transparente & $\begin{array}{l}\text { El azul de Alcián tiñe los GAGs } \\
\text { del blastema de los lepido- } \\
\text { triquias. }\end{array}$ & $\begin{array}{l}\text { La alizarina marca la } \\
\text { calcificación progresiva y el } \\
\text { azul de Alcián tiñe los GAGs } \\
\text { del blastema de los lepido- } \\
\text { triquias. }\end{array}$ \\
\hline
\end{tabular}


II esta aleta está involucionando (Fig. 2) y en el grupo III está ausente (Fig. 3).

En el grupo I la aleta caudal no ha alcanzado su forma definitiva pero ha iniciado la formación de lepidotriquias (Fig. 1). En el grupo 2, la aleta caudal comprende entre 1920 lepidotriquias y se constituyen dos lóbulos uno dorsal y otro ventral, ambos bajo la notocorda. Los rayos de cada lóbulo crecen más rápido que los rayos que se encuentran entre los lóbulos y se forma un surco entre ellos (Fig. 2). En el grupo 3 se observa claramente la aleta bilobulada, se mantienen 19 lepidotriquias pero ahora están en proceso de calcificación además se pueden reconocer con facilidad las articulaciones y bifurcaciones (Fig. 3).

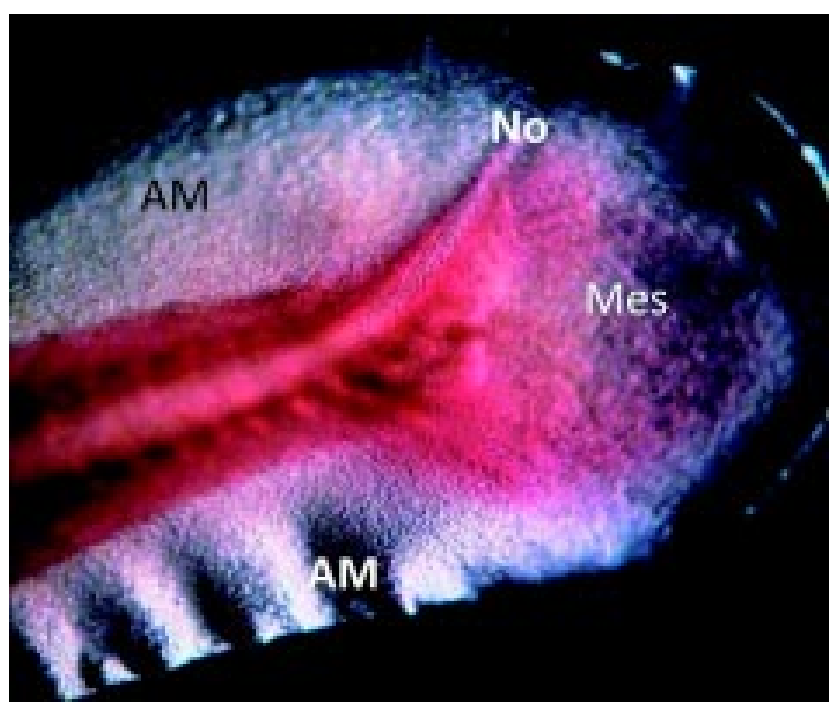

Fig. 1. Grupo 1, presencia de aleta mediana (AM), notocorda (No) mesénquima que forma los rayos bajo la notocorda (Mes). Tinción "in toto" con rojo Sirius. 50X.

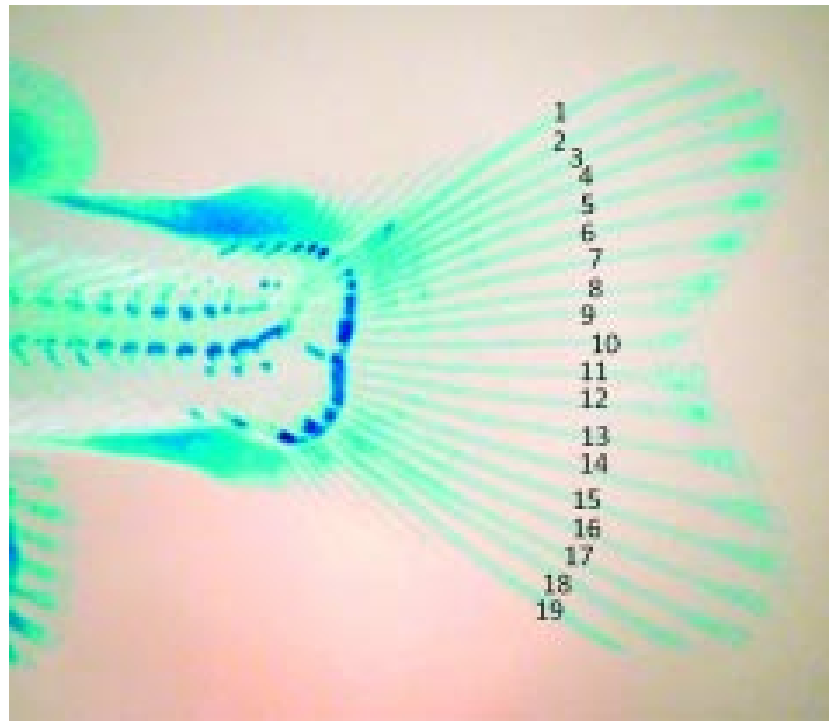

Fig. 2. Grupo 2, 19 lepidotriquias destacadas por azul de Alcián. Técnica de AAA para cartílago y hueso.
Anatomía de la aleta caudal. En el grupo I ( $15 \mathrm{~mm}) \mathrm{La}$ aleta caudal se observa distalmente como un solevantamiento mesenquimático con formación inicial de rayos y revestido por epitelio . Estos rayos no son fácilmente detectables y no presentan segmentaciones ni bifurcaciones, tampoco se evidencian actinotriquias ni melanomacrófagos y no se reconoce lóbulos ni el surco entre lóbulos (Fig. 1) (Tabla I).

En el grupo II (30 mm) se identifican dos lóbulos que tienen un tamaño similar y están separados por un surco, En cada lóbulo se han constituido 9-10 rayos o lepidotriquias y tanto en sus extremos dorsal como ventral se ven lepidotriquias de longitud menor (Figs. 2 y 4). Las lepidotriquias son visibles con el azul de Alcián y no se tiñen con la alizarina (Fig. 4). Cada lepidotriquia esta formada por dos hemirayos simétricos y paralelos que presentan a su vez un promedio de 7 segmentos cortos separados por 6 articulaciones y conectadas una a la otra por ligamentos de colágeno Cada rayo se bifurca en dos en la región más distal de la aleta (Figs. 2 y 4), Los interrayos permanecen transparentes y no se tiñen (Fig. 2) (Tabla I).

En el grupo III $(60 \mathrm{~mm})$ las lepidotriquias se han empezado a calcificar en una progresión que avanza desde proximal a distal (Figs. 3, 5 y 6). Esto es visible externamente por un color rojizo de la alizarina. La parte mas distal de las lepidotriquias se observa de color azul debido al predominio de glicosaminoglicanos. Las

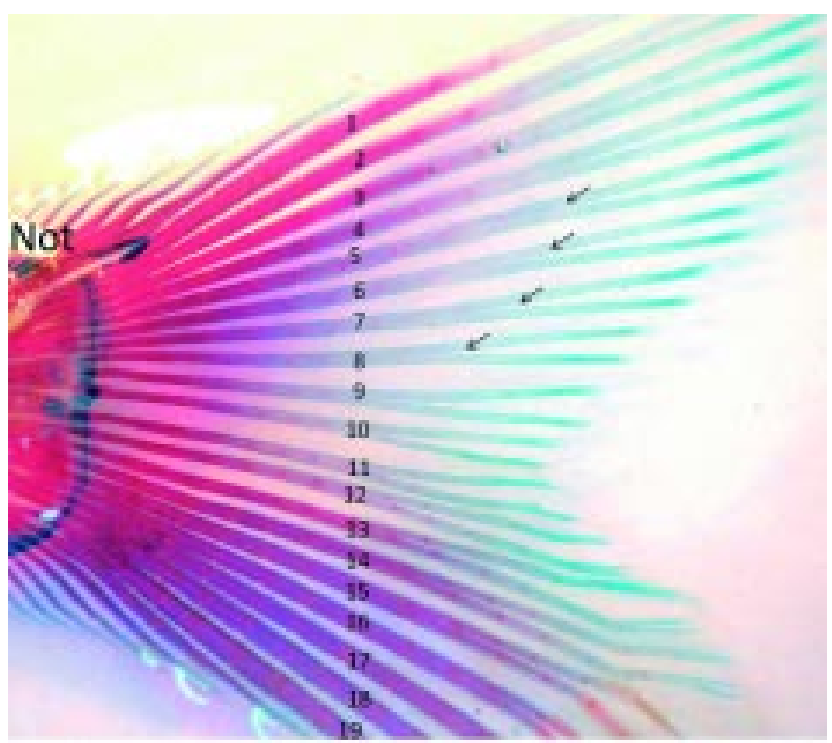

Fig. 3. Grupo 3, 19 lepidotriquias, Se identifica el avance de la osificación desde proximal a distal. Los rayos dorsales y más ventrales se encuentran más avanzados que los mediales. Se identifica además las bifurcaciones de los rayos (flechas). Técnica de AAA. 


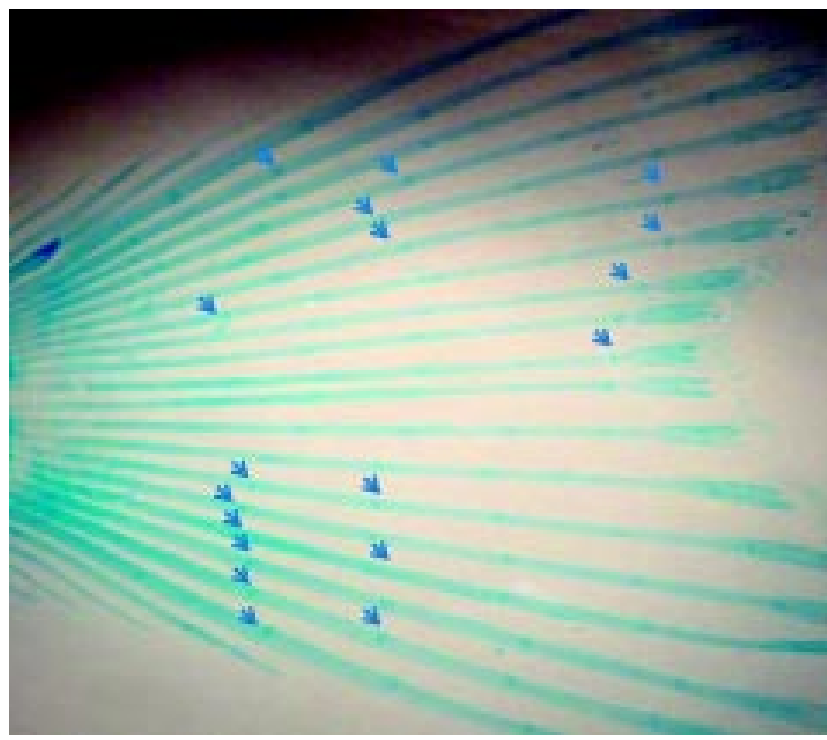

Fig. 4. Grupo 2, articulaciones (flechas) en lepidotriquias. Técnica de AA-A, 30X.

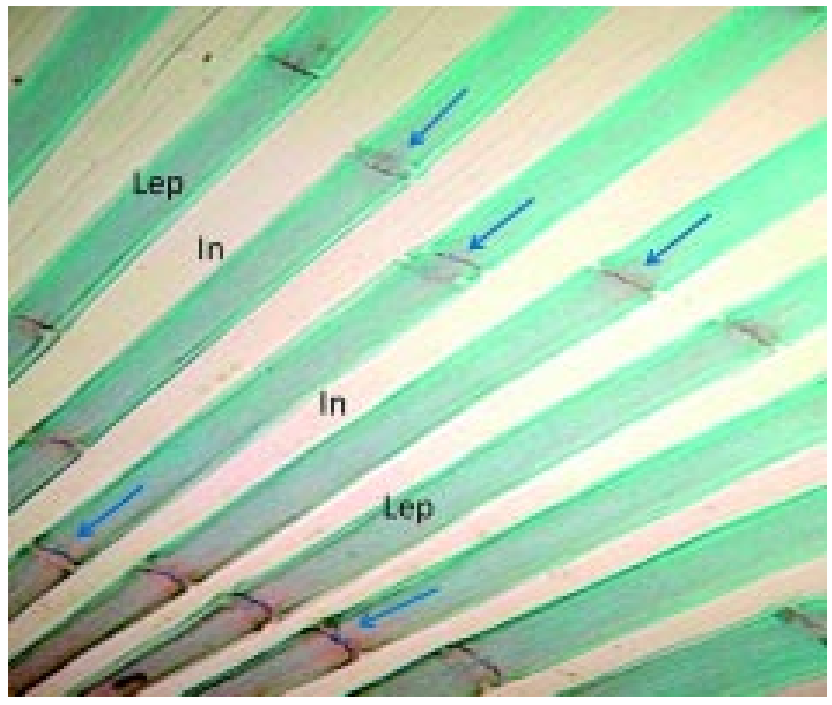

Fig. 5. Grupo 3, articulaciones (flechas) en lepidotriquias (Lep), e inter-rayos (In). Técnica de AAA 50X.

segmentaciones aumentaron a un promedio de 11 debido a que se han formado 10 articulaciones en las lepidotriquias de mayor longitud. En los extremos dorsales y ventrales se evidencian lepidotriquias de reducido tamaño inmersas en una matriz muy positiva al azul de Alcián (Fig. 6). Las bifurcaciones se observan claramente, pero las actinotriquias se observan con dificultad (Tabla I).

Histología de la aleta caudal. La aleta caudal está revestida por un epitelio de revestimiento pluriestratificado constituido por una capa de células basales, células intermedias y superficiales con muchas células caliciformes ricas en GAGs. Entre el epitelio y mesénquima se evi-

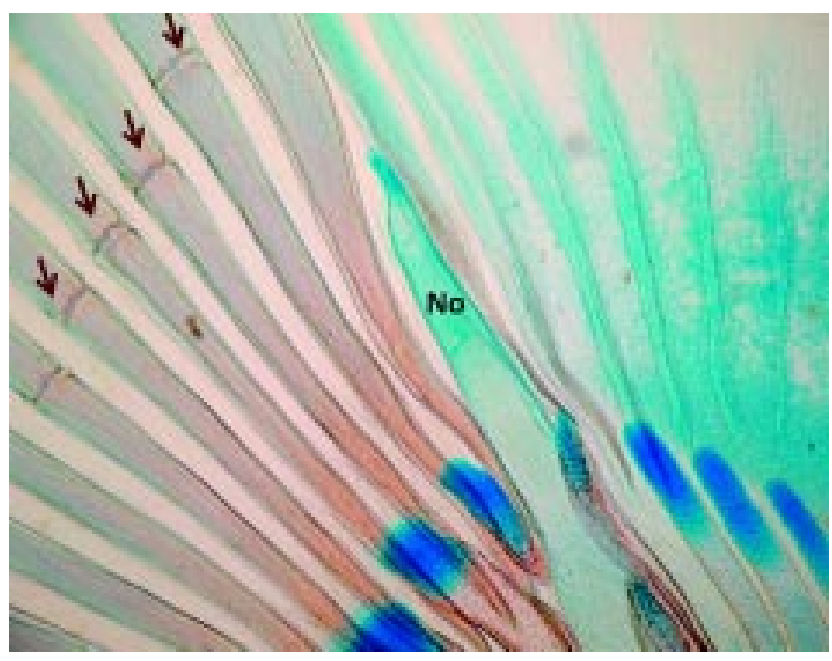

Fig. 6. Grupo 3, lepidotriquias de poco desarrollo dorsales a la notocorda (azules) y lepidotriquias propiamente tales ventrales a la notocorda (No) de color rosado que indica calcificación. Técnica de AA-A 50X.

dencia una membrana basal con colágeno I y III. Este epitelio se invagina formando columnas donde se ubicará las lepidotriquias (Fig. 7). La formación de las lepidotriquias se inicia por lo tanto en contacto con la epidermis y alcanza al interior de la dermis. En los grupos II y III, Los lepidotriquias están inmersas en un mesénquima laxo con características embrionarias (Fig. 7). En la interfase epitelio mesénquima se observa una membrana basal con resfuerzo de fibras colágenas tipo I y III y una matriz lepidotriquial formada por los escleroblastos (Fig. 7).

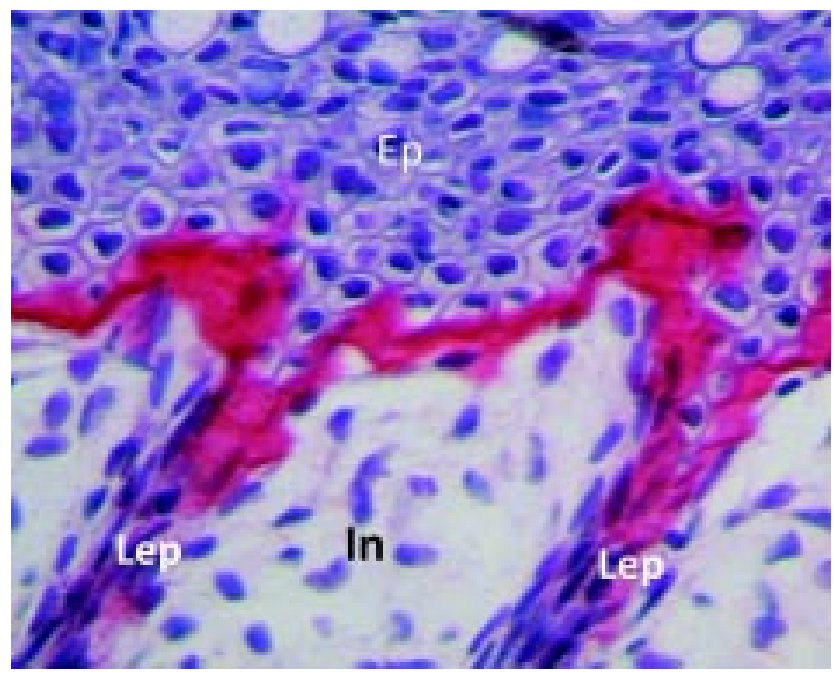

Fig. 7. Grupo 2. A) Región más distal de la aleta caudal. Se observa Epidermis (Ep) y formación inicial de lepidotriquias (Lep). En la interfase epitelio-mesénquima se observa la matriz elaborada por escleroblastos (color rojo) e interrayos (In), Técnica Rojo sirius sin polarizadores. $100 \mathrm{X}$. 
En el grupo III se evidencian bifurcaciones dicotómicas (Fig. 8) con muy poco colágeno tipo I (Fig. 9), en cambio en las articulaciones predomina el colágeno I (Figs. 9 y 10). El área interrayo presenta un tejido mesenquimal prácticamente sin fibras colágenas. Las lepidotriquias tienen un núcleo interno constituído por células mesenquimales un blastema muy rico en GAGs, escasas fibras colágenas, y muchos vasos sanguíneos, lo cual está revestido por tejido óseo que forma dos hemirayos (Fig. 11), Estas lepidotriquias se tiñen de color azul con el colorante azul de Alcián pero esto no corresponde a cartílago hialino, sino que al glicosaminoglicano del blastema que se encuentra al interior (Fig. 12). El hueso está revestido por células aplanadas y cúbicas que corresponden a los escleroblastos (Fig. 12). La calcificación de hueso ocurre lentamente desde la región proximal a la distal (Fig. 3). Los melanóforos se dispersan a través de la aleta caudal (Fig. 13).

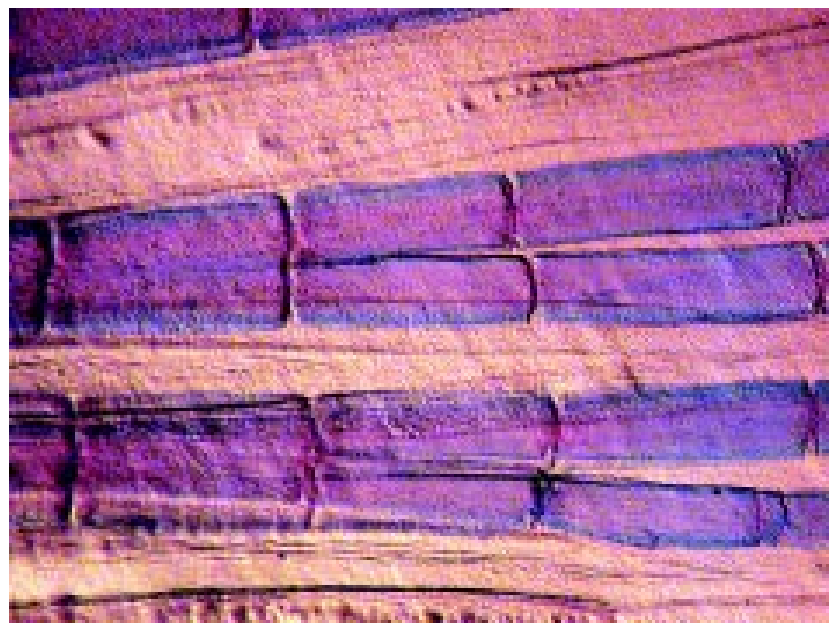

Fig. 8. Grupo 2, bifurcaciones de lepidotriquias, Tecnica de AAA $50 \mathrm{X}$.

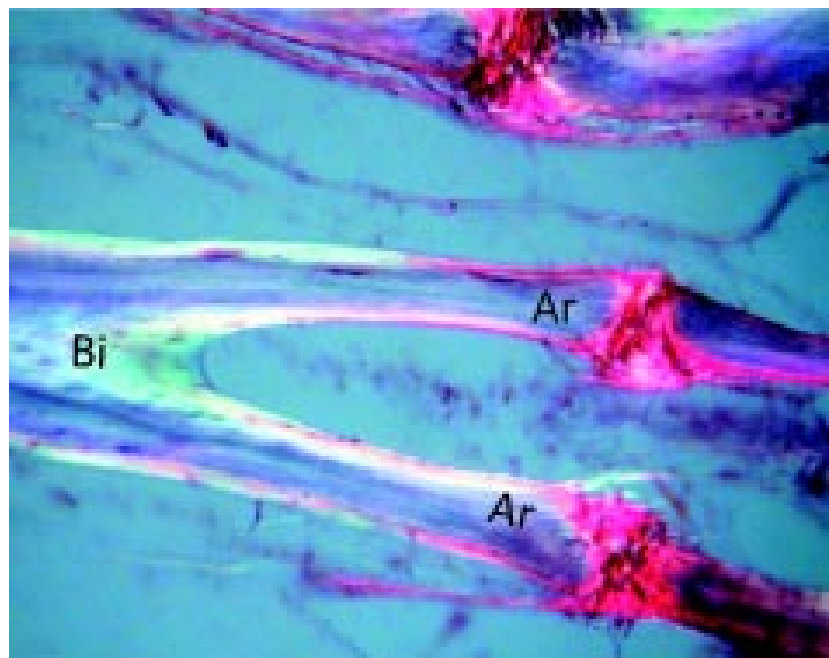

Fig. 9. Bifurcaciones (B) y articulaciones (Ar). Técnica Rojo Sirius de Junqueira. 50X.
Además de las lepidotriquias propiamente tales se pueden observar pequeñas lepidotriquias en las regiones más dorsales y ventrales de la aleta caudal, las cuáles no presentan bifurcaciones ni tampoco actinotriquias (Fig. 14).

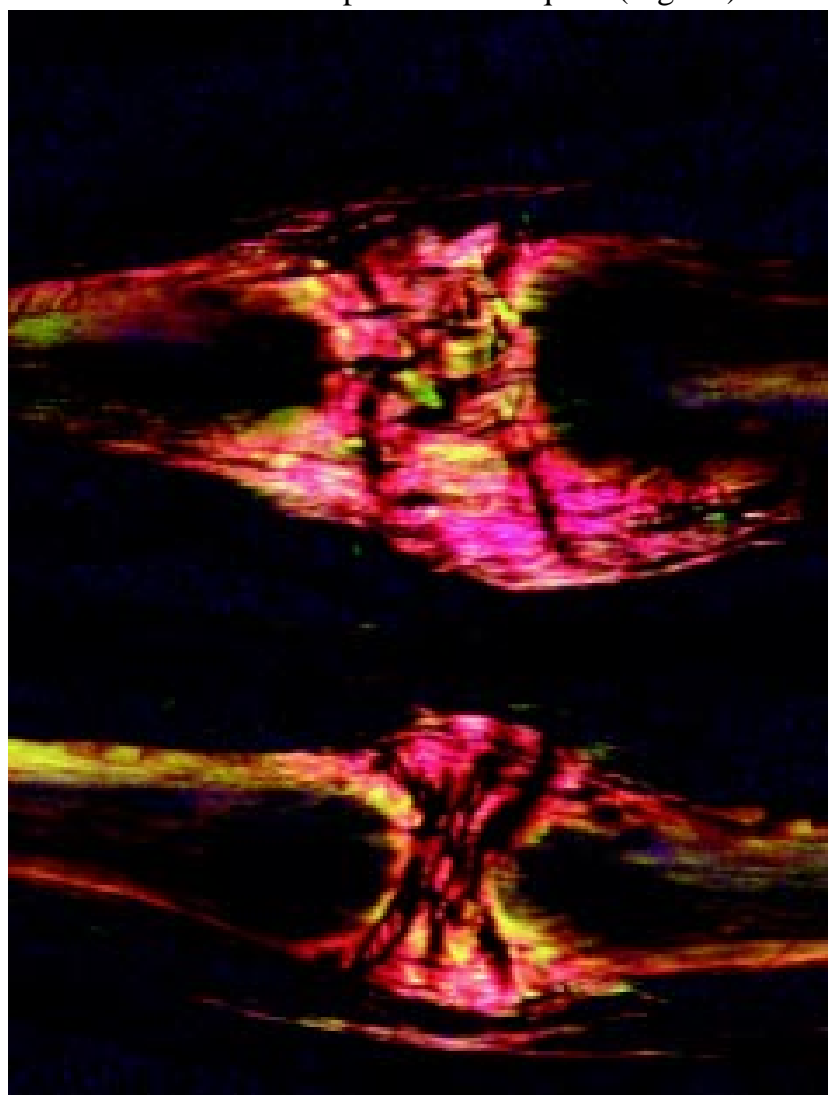

Fig. 10. Articulaciones constituídas principalmente por colágeno I. Técnica Rojo Sirius de Junqueira 400X.

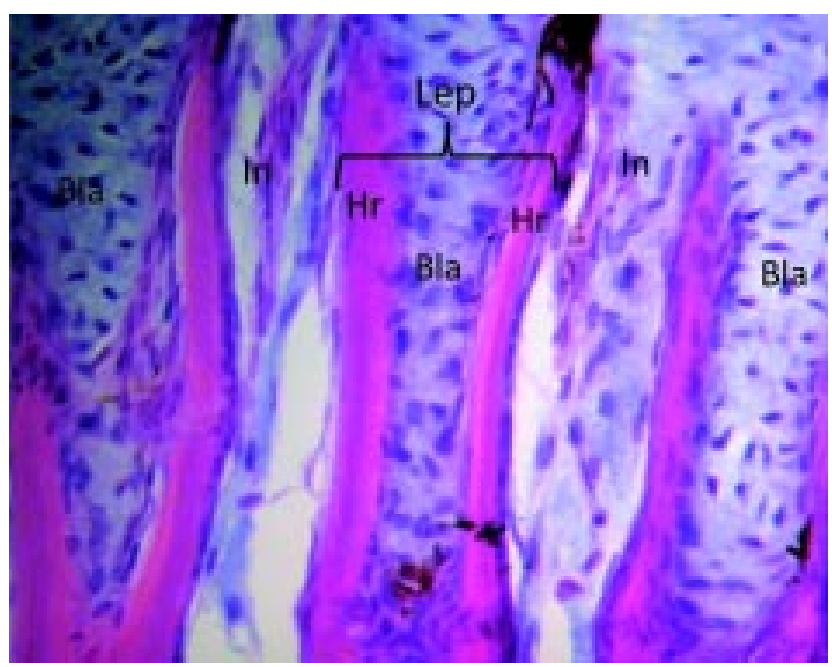

Fig. 11. Grupo 2, corte longitudinal de tres lepidotriquias (Lep) constituido por un blastema de tejido mesenquimal que se tiñe intensamente con azul de Alcián (Bla) y dos semirayos óses simétricos y paralelos (Hr) También se encuentra un blastema interrayo laxo con muchos vasos sanguíneos (In). Técnica H-E- azul de Alcián, 100X. 


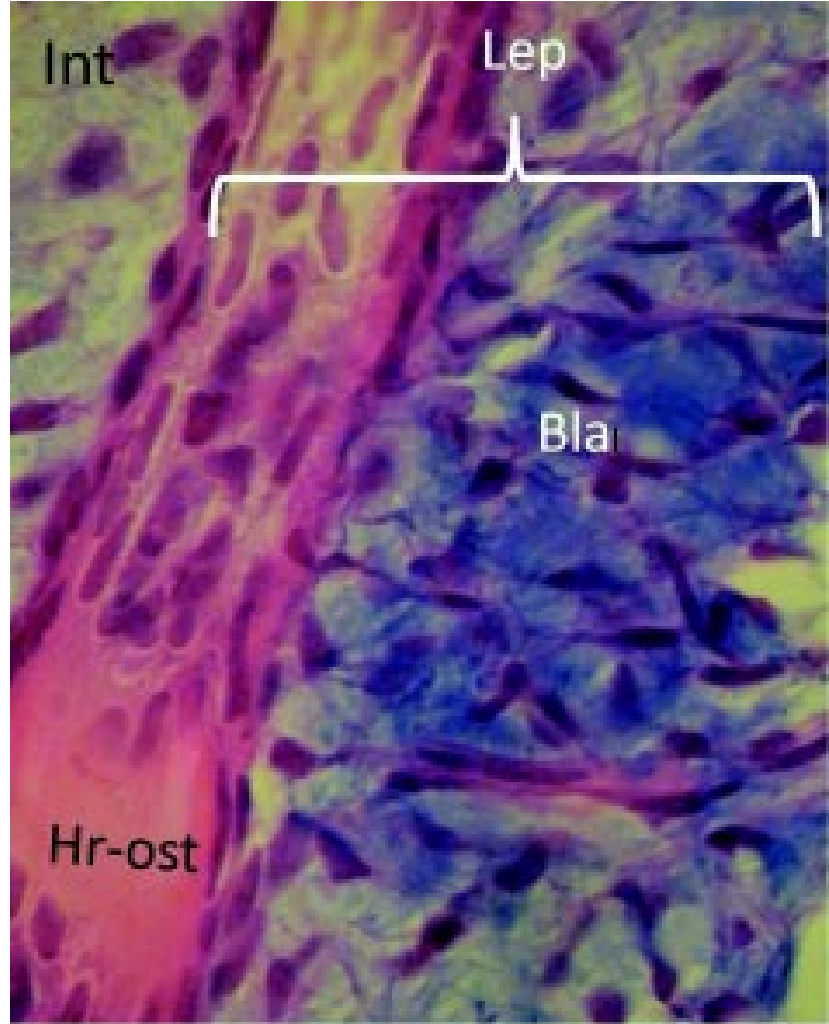

Fig. 12. Hemirayo con escleroblastos (Hr-ost), encerrando una matriz de tejido mesenquimal muy afín al azul de Alcián (Bla). Ambos constituyen una lepidotriquia (Lep). Int: blastema interrayo. Técnica H-E/azul de Alcián, 400X.

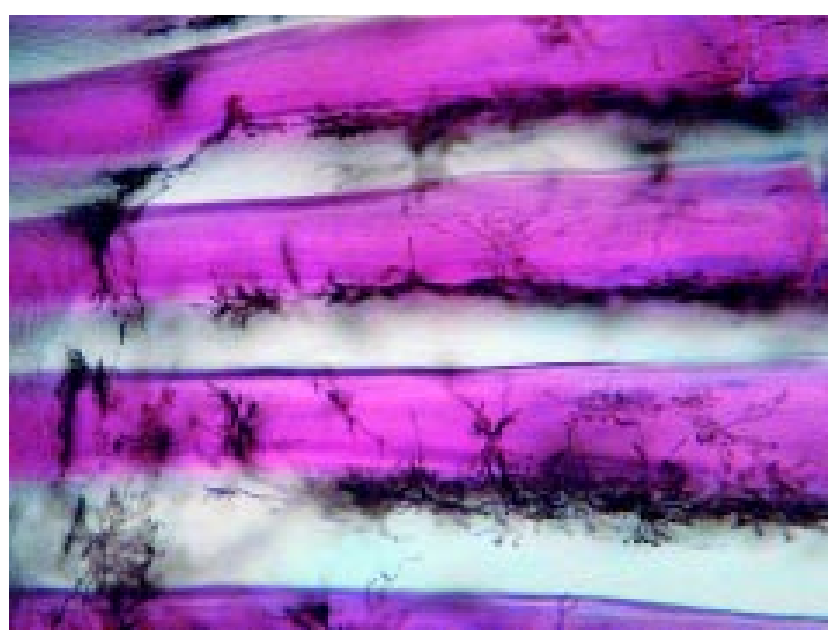

Fig. 13. Grupo 3, lepidotriquias calcificadas y revestidas con melanomacrófagos. Técnica AAA “in toto" 200X.

\section{DISCUSIÓN}

La anatomía de la aleta caudal del salmón en las tres etapas estudiadas es similar a la secuencia que se encuentra en el pez cebra (Rolland-Lagan et al.), sin embargo hay

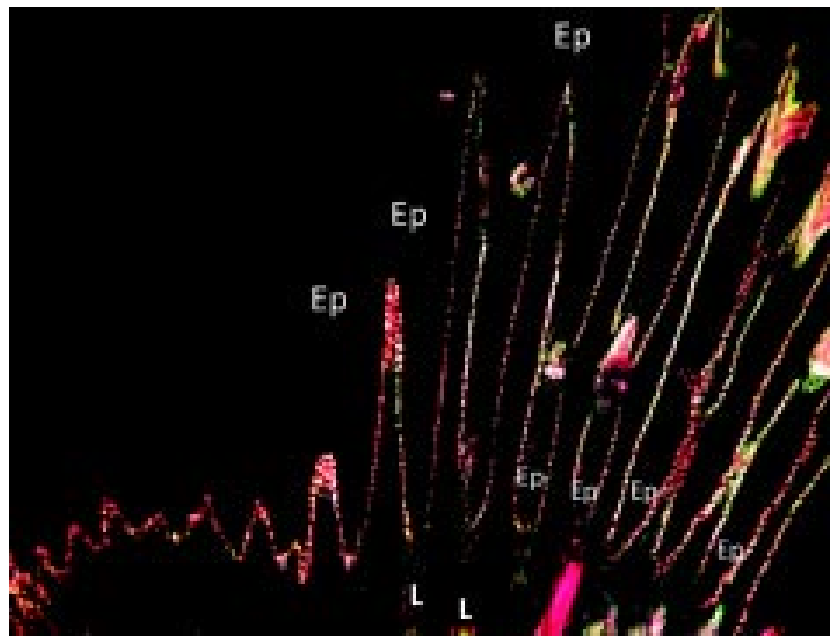

Fig. 14. Grupo 2, variaciones de tamaño de las pequeñas lepidotriquias más dorsales y las propiamente tales. En la interfase epitelio mesénquima se observa una membrana basal con resfuerzo de fibras colágenas I y III. (50X) Rojo Sirius.

importantes diferencias cronológicas entre ambas especies. Los segmentos son mas largos dentro de un rayo en las posiciones proximales que en posiciones distales y los segmentos son mas largos y numerosos en las lepidotriquias que componen los lóbulos comparados con aquellos que se encuentran en el surco interlobar, esto es similar a lo observado en pez cebra por Iovine \& Johnson (2000).

La piel que reviste la aleta caudal del alevín de salmón está constituída por epidermis de epitelio estratificado y dermis constituida por tejido con características mesenquimales. El análisis morfológico nos permite describir que existe una relación entre la capa basal de la epidermis y la formación de las lepidotriquias. Esto está de acuerdo con los estudios sobre regeneración de la epidermis en pez cebra y de carpa adultos realizadas por Akimenko et al. $(1995,2003)$ y Böckelmann $e t$ al. y que indican que la epidermis tiene un papel fundamental en el proceso de formación de lepidotriquias.

Debido a que la piel de los peces es vital como defensa frente al ambiente externo (Quilhac \& Sire, 1999) el tiempo de regeneración de la piel debe ser muy rápido para mantener la protección frente a hongos, bacterias y protozoos. La presencia de esta capa epidérmica es importante con el fin de estimular la regeneración de otras estructuras perdidas de la aleta (Becerra et al., 1996; Bechara et al., 2000, 2003; Böckelmann \& Bechara, 2004). Internamente se observan 19 rayos elongados de origen dérmico que corren de proximal a distal en la aleta caudal, esto es diferente a lo descrito por Akimenko et al. (2003) para pez cebra que reporta un promedio de 15-18 lepidotriquias. Cada 
lepidotrichia se compone de dos hemirayos opuestos y cóncavos con tejido mesenquimatico intra-rayo central y está de acuedo a lo observado por Montes et al. (1982) en el pez adulto. Con azul de Alcián se reconoce los glicosaminoglicanos presentes en el mesénquima en todas las regiones del blastema, sin embargo, el blastema mas periférico se relaciona con células formadoras de lepidotriquias. Los escleroblastos se encuentran en los compartimentos laterales donde ellos secretan directamente matriz ósea, esto es similar a lo observado por Santamaria et al. (1992). Las articulaciones consisten en huesos de extremos redondeados que se enfrentan entre sí. El compartimento mesenquimal sigue siendo continua y la articulación física se produce sólo en la matriz ósea. Esto es consistente con la caracterización previa de las articulaciones como "fibroso" (Borday et al., 2001), y con las definiciones que dicen que las articulaciones están conectadas por tejido conectivo (Iovine, 2007). En este estudio no se observaron con facilidad las espículas fusiformes llamados actinotriquias. Esto es diferente a lo observado por (Böckelmann \& Bechara, 2009) quienes observaron que después de la amputación de la cola son estas estructuras las que muestran una capacidad regenerativa extremádamente rápida

Bechara et al. (2003) observaron que la aspirina, un antiinflamatorio no esteroidal inhibe la formación de actinotrichia, y esto sugiere que su inhibición se puede deber a que la aspirina probablemente interfiere con la vía de señales Sonic hedgehog (Shh). Böckelmann \& Bechara (2009) observaron que durante el proceso de regeneración de actinotrichia, naproxeno, no interfiere con la síntesis de actinotrichia durante el proceso regenerativo de la aleta caudal. Esto puede ocurrir porque naproxen no influye en la expresión de genes requeridos para el proceso de regeneración, tales como Shh el cual está involucrado en la formación de actinotrichia. El blastema inter-rayos es laxo, también tiene apariencia mesenquimal y muchos vasos sanguíneos. similar a lo que ocurre en pez cebra (Danio rerio Hamilton-Buchanan). Murciano et al. $(2002,2007)$ han presentado evidencia que sugiere que el blastema interrayo es necesario para una bifurcación adecuada de cada rayo durante la regeneración. El tejido circundante y la epidermis interrayo pueden modular la longitud del rayo.

El grupo A, la aleta caudal está poco desarrollada, las lepidotriquias son del mismo tamaño, por lo tanto no se han constituido los lóbulos, esto se relaciona con el estudio de Hernández et al. (2013), que indica que no hay movimientos natatorios de los peces en esta fase post-eclosional debido a que durante esta época los alevines con saco vitelino, permanecen en el fondo del tanque. Según estos autores al momento de la eclosión, el sistema nervioso está muy indiferenciando, $\mathrm{y}$ durante las primeras semanas de vida del alevín ocurre la diferenciación de las neuronas y neurogénesis (Hernández \& Rojas, 2013). En el grupo B, las lepidotriquias han crecido en forma diferente y se constituyen dos lóbulos de similar tamaño. Cuando quedan muy pocos nutrientes en el saco vitelino, los peces se elevan a la superficie y se adhieren a las plantas. En el grupo C las lepidotriquas han crecido en longitud y se puede reconocer los segmentos, las bifurcaciones y el inicio de la calcificación. Los peces nadan con facilidad. Este estudio permite un mayor conocimiento de la histogénesis normal de la aleta caudal en fase de alevinaje facilitando el diagnóstico de patologías y aporta información para estudios comparativos entre la ontogenia de la aleta caudal y la regeneración después de la amputación total de la cola en salmones.

ROJAS, M.; PELLÓN, M. \& DEL SOL, M. Salmon caudal fin development (Salmo salar.). Int. J. Morphol., 33(2):514-521, 2015.

SUMMARY: Caudal fin pathologies and traumas can affect swimming, impede food and exhaust efficiency, and also increase susceptibility to bacterial and fungal infections. Adult salmon can regenerate their fin quickly and completely if it is amputated. However, yolk sac fry expressing anatomical defects in the caudal fin have been reported in southern Chile and are associated to a high mortality rate where regeneration does not occur. There are many studies on adult salmon but this description does not match the morphology of the juvenile phase. We describe the anatomy and histology of the caudal fin in salmon $15 \mathrm{~mm}, 30 \mathrm{~mm}$ and $60 \mathrm{~mm}$ to facilitate the early diagnosis of diseases of the caudal fin. We worked with 60 salmon divided into three groups of 20 in steps of 15, 30 and $60 \mathrm{~mm}$. 10 salmon from each group were processed with Hanken \& Wassersug anatomical techniques. Another 10 fry from each group were processed using H\&E/Alcian blue $\mathrm{pH} 2.5$ techniques: for glycosaminoglycans and technical histochemistry Picrosirius Junqueira for collagen I and III. Upon hatching of fish (group 1) the caudal fin has no definitive form but has commenced training ray or lepidotriquias. In group 2, the caudal fin comprises from 19 to 20 lepidotriquias and two lobes one dorsal and one ventral, both are constituted under the notochord. Each lobe ray grows faster than the rays that lie between the lobes and a groove is formed between them. In group $3 \mathrm{clearly}$ shows the bilobed flap, 19 lepidotriquias that are now in the process of calcification are maintained. Each lepidotriquia grows distally by forming joints and segments. In group 2 an average of 4-5 lepidotriquia joints were recorded and in group 3 there was an increase at 610 joints. This description of normal fry flap facilitates comparative study of the deformed fin.

KEY WORDS: Development; Caudal fin; Lepidotrichia; Juvenile salmon; Salmo salar. 


\section{REFERENCIAS BIBLIOGRÁFICAS}

Akimenko, M. A.; Johnson, S. L.; Westerfield, M. \& Ekker, M. Differential induction of four msx homeobox genes during fin development and regeneration in zebrafish. Development., 121(2):347-57, 1995.

Akimenko, M. A.; Marí-Beffa, M.; Becerra, J. \& Géraudie, J. Old questions, new tools, and some answers to the mystery of fin regeneration. Dev. Dyn., 226(2):190-201, 2003.

Becerra, J.; Montes, G. S.; Bexiga, S. R. \& Junqueira, L. C. Structure of the tail fin in teleosts. Cell Tissue Res., 230(1):127-37, 1983.

Becerra, J.; Junqueira, L. C.; Bechara, I. J. \& Montes, G. S. Regeneration of fin rays in teleosts: a histochemical, radioautographic, and ultrastructural study. Arch. Histol. Cytol., 59(1):15-35, 1996.

Bechara, I. J.; Joazeiro, P. P.; Marí-Beffa, M.; Becerra, J. \& Montes, G. S. Collagen-affecting drugs impair regeneration of teleost tail fins. J. Submicrosc. Cytol. Pathol., 32(2):273-80, 2000.

Bechara, I. J.; Böckelmann, P. K.; Montes, G. S. \& Cruz-Höfling, M. A. Inhibition of caudal fin actinotrichia regeneration by acetylsalicylic acid (aspirin) in teleosts. Braz. J. Morphol. Sci., 20(2):67-74, 2003.

Böckelmann, P. K. \& Bechara, I. J. Effect of naproxen on tail fin regeneration in teleost. J. Submicrosc. Cytol. Pathol., 36(1):5564, 2004.

Böckelmann, P. K. \& Bechara, I. J. The regeneration of the tail fin actinotrichia of carp (Cyprinus carpio, Linnaeus, 1758) under the action of naproxen. Braz. J. Biol., 69(4):1165-72, 2009.

Böckelmann, P. K.; Ochandio, B. S \& Bechara, I. J. Histological study of the dynamics in epidermis regeneration of the carp tail fin (Cyprinus carpio, Linnaeus, 1758). Braz. J. Biol., 70(1):21723, 2010.

Borday, V.; Thaëron, C.; Avaron, F.; Brulfert, A.; Casane, D.; Laurenti, P. \& Géraudie, J. evx1 transcription in bony fin rays segment boundaries leads to a reiterated pattern during zebrafish fin development and regeneration. Dev. Dyn., 220(2):91-8, 2001.

Hanken, J. \& Wassersug, R. The visible skeleton. A new doublestain technique reveals the native of the 'hard' tissues. Funct. Photogr., 16:22-6, 1981.

Hernández, H. \& Rojas, M. Development of Spinal Cord of the Salmon (Salmo salar) during the period post eclosional. Int. J. Morphol., 31(1):172-6, 2013.

Iovine, M. K. Conserved mechanisms regulate outgrowth in zebrafish fins. Nat. Chem. Biol., 3(10):613-8, 2007.
Iovine, M. K. \& Johnson, S. L. Genetic analysis of isometric growth control mechanisms in the zebrafish caudal Fin. Genetics, 155(3):1321-9, 2000.

Junqueira, L. C.; Bignolas, G. \& Brentani, R. R. Picrosirius staining plus polarization microscopy, a specific method for collagen detection in tissue sections. Histochem. J., 11(4):447-55, 1979.

Marí-Beffa, M.; Palmqvist, P; Marín-Girón, F.; Montes, G. S. \& Becerra, J. Morphometric study of the regeneration of individual rays in teleost tail fins. J. Anat., 195(Pt. 3):393-405, 1999.

Marí-Beffa, M. \& Murciano, C. Dermoskeleton morphogenesis in zebrafish fins. Dev. Dyn., 239(11):2779-94, 2010.

Montes, G. S.; Becerra, J.; Toledo, O. M.; Gordilho, M. A. \& Junqueira, L. C. Fine structure and histochemistry of the tail fin ray in teleosts. Histochemistry, 75(3):363-76, 1982.

Murciano, C.; Fernández, T. D.; Durán, I.; Maseda, D.; Ruiz-Sánchez, J.; Becerra, J.; Akimenko, M. A. \& Marí-Beffa, M. Ray-interray interactions during fin regeneration of Danio rerio. Dev. Biol., 252(2):214-24, 2002.

Murciano, C.; Pérez-Claros, J.; Smith, A.; Avaron, F.; Fernández, T. D.; Durán, I.; Ruiz-Sánchez, J.; García, F.; Becerra, J.; Akimenko, M. A. \& Marí-Beffa, M. Position dependence of hemiray morphogenesis during tail fin regeneration in Danio rerio. Dev. Biol., 312(1):272-83, 2007.

Quilhac, A. \& Sire, J. Y. Spreading, proliferation, and differentiation of the epidermis after wounding a cichlid fish, Hemichromis bimaculatus. Anat. Rec., 254(3):435-51, 1999.

Rolland-Lagan, A. G.; Paquette, M.; Tweedle, V. \& Akimenko, M. A. Morphogen-based simulation model of ray growth and joint patterning during fin development and regeneration. Development, 139(6):1188-97, 2012.

Santamaría, J. A.; Marí-Beffa, M. \& Becerra, J. Interactions of the lepidotrichial matrix components during tail fin regeneration in teleosts. Differentiation, 49(3):143-50, 1992.

Stoick-Cooper, C. L.; Moon, R. T. \& Weidinger, G. Advances in signaling in vertebrate regeneration as a prelude to regenerative medicine. Genes Dev., 21(11):1292-315, 2007.

\section{Dirección para Correspondencia: \\ Mariana Rojas Rauco \\ Programa de Anatomía y Biología del Desarrollo \\ Universidad de Chile \\ Independencia 1027 \\ Santiago \\ CHILE}

Email: mrojas@med.uchile.cl

Recibido : 11-01-2015

Aceptado: 27-03-2015 